\title{
Roughness-Enhanced Reliability of MOS Tunneling Diodes
}

\author{
C.-H. Lin, Student Member, IEEE, F. Yuan, Student Member, IEEE, C.-R. Shie, K.-F. Chen, B.-C. Hsu, M. H. Lee, \\ W. W. Pai, and C. W. Liu, Senior Member, IEEE
}

\begin{abstract}
Both electrical and optical reliabilities of PMOS and NMOS tunneling diodes are enhanced by oxide roughness, prepared by very high vacuum prebake technology. For the rough PMOS devices, as compared to the flat PMOS devices, the Weibull plot of $T_{B D}$ shows 2.5 -fold enhancement at $63 \%$ failure rate, while both the $\mathrm{D}_{2}$ and $\mathrm{H}_{2}$-treated flat PMOS devices show similar inferior reliability. For the rough NMOS devices, as compared to the flat NMOS devices, the Weibull plot of $T_{B D}$ shows 4.9-fold enhancement at $63 \%$ failure rate. The time evolutions of the light emission from the rough PMOS and NMOS diodes degrade much less than those of the flat PMOS and NMOS diodes. The momentum reduction perpendicular to the $\mathrm{Si} / \mathrm{SiO}_{2}$ interface by roughness scattering could possibly make it difficult to form defects in the bulk oxide and at the $\mathrm{Si} / \mathrm{SiO}_{2}$ interface by the impact of the energetic electrons and holes.
\end{abstract}

Index Terms-Electroluminescence, MOS, reliability, roughness, ultrathin oxide.

\section{INTRODUCTION}

$\mathbf{T}$ HE SILICON dioxide in MOSFETs plays a major role in the electrical properties of such devices. As the oxide thickness continues to scale down, the roughness of the gate oxide is no longer negligible. Several groups have reported the effects of interface roughness on the electrical performance. The interface roughness degraded MOSFET channel mobility due to the surface scattering [1], [2], degraded the time-dependent dielectric breakdown (TDDB) characteristics of MOS capacitors, and increased the tunneling current through the oxide [3]. On the other hand, the advantage of oxide roughness has also been utilized to enhance the electroluminescence efficiency in the MOS tunneling diodes [4], but the oxide roughness has not been reportedly beneficial to the electrical properties of MOS devices. The deuterium enhanced oxide reliability has been investigated by many groups for years [5]-[7], but no isotope effect has been observed in the deuterium-treated MOS devices stressed in the hole injection condition [8]-[10]. The tunneling-hole-induced traps in bulk oxide are responsible for this soft breakdown, and oxide degrades even with the deuterium passivation [10], [11].

Manuscript received February 20, 2002; revised April 29, 2002. This work was supported by the National Science Council of Taiwan, R.O.C., (Contracts 90-2212-E-002-226 and 90-2215-E-002-034), and the Institute of Applied Science and Engineering Research, Academia Sinica, Taipei, Taiwan. The review of this letter was arranged by Editor C.-P. Chang.

C.-H. Lin, F. Yuan, C.-R. Shie, K.-F. Chen, B.-C. Hsu, M. H. Lee, and C. W. Liu are with the Graduate Institute of Electronics Engineering and the Department of Electrical Engineering, National Taiwan University, Taipei, Taiwan, R.O.C. (e-mail: chee@cc.ee.ntu.edu.tw).

W. W. Pai is with the Center for Condensed Matter Science, National Taiwan University, Taipei, Taiwan, R.O.C.

Publisher Item Identifier S 0741-3106(02)05983-9.
Therefore, it is of great importance and interest to improve the reliability of ultrathin gate oxide in a hole-stressed device. In this letter, we investigate the oxide roughness effect on the reliability of PMOS tunneling diodes and demonstrate that the rough oxide can enhance both the electrical and optical reliability of PMOS tunneling diodes. The same results are also observed in the NMOS tunneling diodes.

\section{GRowTH AND DEVICE FABRICATION}

The ultrathin gate oxide was grown by rapid thermal oxidation (RTO) on 1-10 $\Omega-\mathrm{cm} \mathrm{n/p-type} \mathrm{silicon} \mathrm{wafer} \mathrm{at} \mathrm{the} 850{ }^{\circ} \mathrm{C}$. The gas flows were $500 \mathrm{sccm}$ nitrogen and $500 \mathrm{sccm}$ oxygen at a reduced pressure. After a HF dip, the wafers were in-situ baked in the very high vacuum condition $\left(<3 \times 10^{-6}\right.$ torr, maintained by a turbo pump) at $1000{ }^{\circ} \mathrm{C}$ to obtain the rough Si surface [12], and then the wafers were baked in hydrogen at $1000{ }^{\circ} \mathrm{C}$ for $1 \mathrm{~min}$ before the rapid thermal oxidation. Note that this very high vacuum prebake should also yield a clean silicon surface [13]. After the growth of ultrathin oxide, the sample was in situ annealed in nitrogen for $10 \mathrm{~min}$ at $900^{\circ} \mathrm{C}$. The oxide thickness was measured by ellipsometry. Due to the roughness on the oxide surface and at oxide/Si interface, the thickness of rough oxide cannot be well defined if there is small deviation from conformal growth model. The electrical thickness by tunneling current or capacitance-voltage $(C-V)$ method cannot be measured accurately due to the large effective area on the rough oxide, and the two-dimensional (2-D) electrical effect in the rough oxide [14], which is unknown. The meaningful thickness of rough oxide itself is an issue to be further investigated. Here, we simply use the thickness measured from ellipsometry as a reference. The roughness was measured by atomic force microscope (AFM) and X-ray reflectivity. The interface roughness was measured by AFM right after HF dip to remove the oxide. The roughness magnitude of the oxide surface is very similar to that of the oxide/silicon interface. This confirms the conformal growth of the oxide [15]. The MOS tunneling diodes were evaporated with $\mathrm{Al}$ gate electrodes with different circular areas defined by photolithography.

\section{RESULTS AND DISCUSSION}

Fig. 1 shows the time evolutions of gate tunneling current for the flat and the rough PMOS tunneling diodes with the oxide thickness of $2.7 \mathrm{~nm}$ and $2.8 \mathrm{~nm}$, respectively, under constant voltage stress (CVS) at gate voltage $\left(V_{g}\right)$ of $2 \mathrm{~V}$. The area size of the devices is $3 \times 10^{-4} \mathrm{~cm}^{2}$. As oxide scaling down, the mechanism of oxide degradation is dominated by the soft breakdown, 


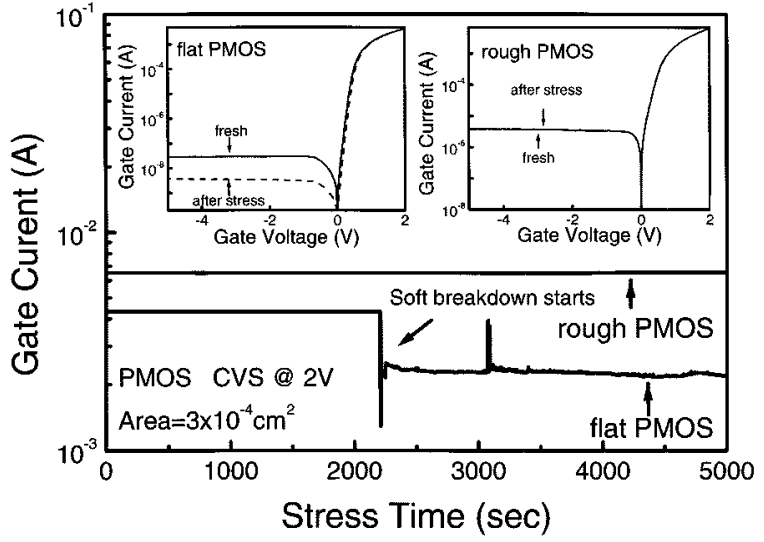

Fig. 1. Time evolutions of gate current of the flat and the rough PMOS tunneling diodes under $2 \mathrm{~V}$ constant voltage stress. The insets are $I-V$ curves before and after stress (left: the flat device, right: the rough device).

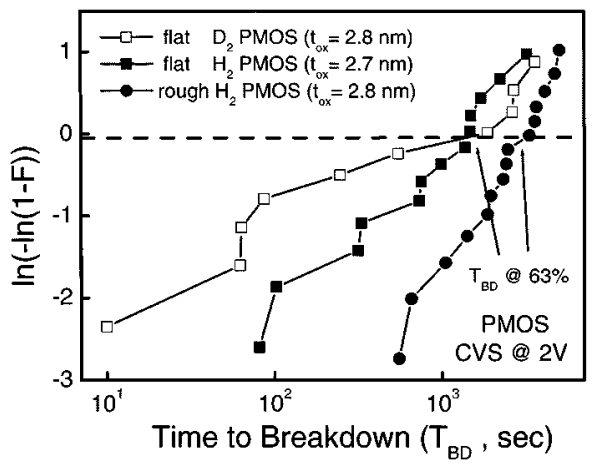

Fig. 2. Weibull plot of the $T_{B D}$ characteristics for the flat $\left(H_{2}, D_{2}\right)$ and the rough $\left(H_{2}\right)$ PMOS tunneling diodes under CVS at $V_{g}=2 \mathrm{~V}$.

which is characterized by the increase of the gate noise [16]. In our case, the breakdown criterion is that the gate current fluctuation (spike) occurs during the CVS as indicated in Fig. 1. The flat PMOS device with the oxide roughness of $0.15 \mathrm{~nm}$ reveals degradation after $\sim 2200$ s stress, while the rough PMOS devices with the oxide roughness of $1.46 \mathrm{~nm}$ shows very little gate current fluctuation under the same stress condition. Note that the rough device has larger injection current density than the flat device probably due to the 2-D electrical effect and the large effective area at $\mathrm{Si} / \mathrm{SiO}_{2}$ interface. Similar results have been observed in [3]. The current-voltage $(I-V)$ characteristics before and after stress are shown in the insets of Fig. 1. The gate current of the flat PMOS device shows a large disparity between the fresh device and the stressed device (left inset). The gate tunneling current in the rough PMOS device remains almost the same after stress (right inset). This indicates that the rough PMOS device has better oxide reliability than the flat PMOS devices.

Fig. 2 shows the Weibull plot of the time to breakdown $\left(T_{B D}\right)$ characteristics for both the flat $\left(H_{2}\right.$ and $D_{2}$-treated) and the rough $\left(H_{2}\right.$-treated) PMOS tunneling diodes under CVS at $V_{g}=2 \mathrm{~V}$. For the rough $\mathrm{H}_{2}$-treated device, as compared to the flat $H_{2}$-treated device, the Weibull plot of $T_{B D}$ shows 2.5-fold enhancement at $63 \%$ failure rate, while both the flat $D_{2}$ and $\mathrm{H}_{2}$-treated PMOS devices show similar inferior reliability. The Weibull plot of charge to breakdown $\left(Q_{B D}\right)$ also shows

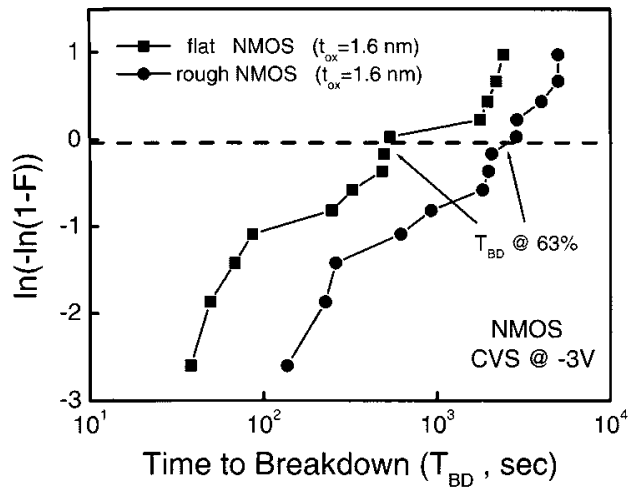

Fig. 3. Weibull plot of the $T_{B D}$ characteristics for the flat $\left(H_{2}\right)$ and the rough $\left(H_{2}\right)$ NMOS tunneling diodes under CVS at $V_{g}=-3 \mathrm{~V}$.

5.7-fold enhancement at $63 \%$ failure rate (not shown here). It is shown that deuterium cannot improve the oxide degradation of the PMOS devices under the hole injection stress [10]. Two kinds of current components exist in the PMOS tunneling diodes. The electron current tunnels from $\mathrm{Si}$ conduction band to $\mathrm{Al}$ and the hole current tunnels from $\mathrm{Al}$ to $\mathrm{Si}$ valence band. The hole tunneling from $\mathrm{Al}$ to $\mathrm{Si}$ can be trapped in the oxide directly and/or break the $\mathrm{Si}-\mathrm{H}$ bonds at $\mathrm{Si} / \mathrm{SiO}_{2}$ interface. Even in the direct tunneling, the tunneling hole can still have excessive energy with respect to the valance band edge. This energy can break the $\mathrm{Si}-\mathrm{H}(\mathrm{D})$ bonds [8]. The electron tunneling from $\mathrm{Si}$ to $\mathrm{Al}$ cannot damage the $\mathrm{Si} / \mathrm{SiO}_{2}$ interface, since there is no excess electron energy at the $\mathrm{Si} / \mathrm{SiO}_{2}$ interface. In the PMOS devices with rough oxide, the injected holes are scattered by the rough $\mathrm{Si} / \mathrm{SiO}_{2}$ and $\mathrm{Al} / \mathrm{SiO}_{2}$ interfaces, and thus the hole momentum perpendicular to the interface is reduced. The perpendicular hole momentum seems crucial for the direct hole trapping in the oxide and the breakage of $\mathrm{Si}-\mathrm{H}$ bonds at $\mathrm{Si} / \mathrm{SiO}_{2}$ interface. The reduction of this momentum prevents the defect formation in the bulk oxide and at the $\mathrm{Si} / \mathrm{SiO}_{2}$ interface, and leads to enhanced reliability in the rough devices. The rough oxide may be an alternative to improve the oxide reliability during the hole injection. Fig. 3 shows the Weibull plot of $T_{B D}$ characteristics for both the $\mathrm{H}_{2}$-treated flat and rough NMOS tunneling diodes with the same oxide thickness of $1.6 \mathrm{~nm}$, under CVS at $V_{g}=-3 \mathrm{~V}$. For the rough device, as compared to the flat device, the Weibull plots of $T_{B D}$ and $Q_{B D}\left(Q_{B D}\right.$ plot not shown here) shows 4.9 and 2.9-fold enhancement, respectively, at $63 \%$ failure rate. The rough NMOS tunneling diodes also reveal improved electrical reliability than the flat NMOS tunneling diodes. Previous works on the voltage acceleration characteristics were based on uniform electric field across the oxide. However, for the rough oxide, the electric field in the oxide is 2-D, and not uniform in the oxide. The maximum electric field in the rough oxide can be larger than that in the flat oxide even at lower gate voltage [14]. The voltage acceleration factor is complicated and still under investigation.

To further investigate the roughness effect on the oxide reliability, the light emission intensity at bandgap energy is measured. The detailed mechanism can be found in [17]. The time evolutions of emission intensity from the flat and the rough PMOS diodes are shown in Fig. 4. The operation gate current is $100 \mathrm{~mA}$ and the device area is $1.5 \times 10^{-2} \mathrm{~cm}^{2}$. The intensity of 


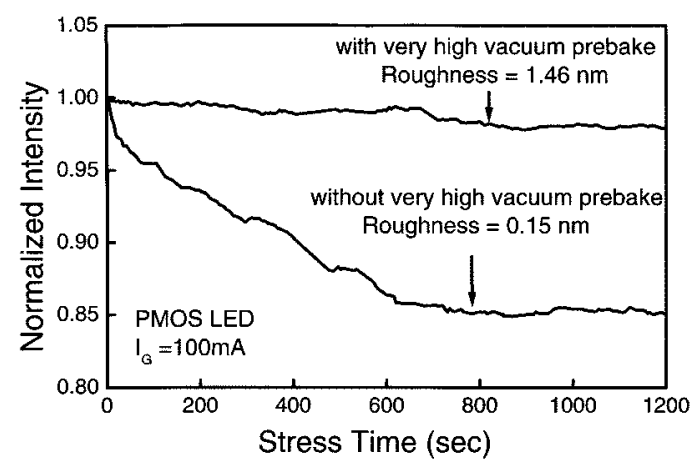

Fig. 4. Time evolutions of light emission intensity from the flat and the rough PMOS diodes. The rough PMOS diode shows much less emission intensity degradation than the flat PMOS device.

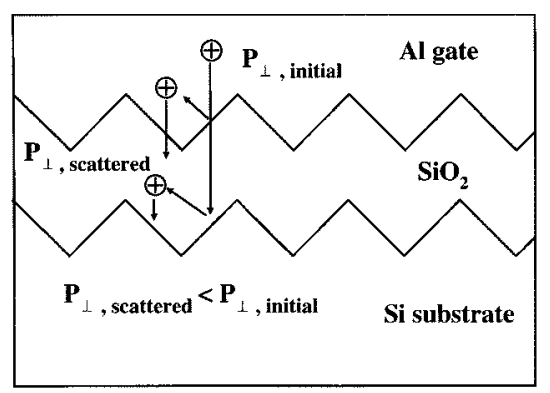

Fig. 5. Illustration of the speculative mechanism of the roughness-enhanced reliability. The hole impact energy perpendicular to the $\mathrm{Si} / \mathrm{SiO}_{2}$ and $\mathrm{Al} / \mathrm{SiO}_{2}$ interface is reduced due to the surface and interface roughness scattering. The multiple scattering of the tunneling hole are possible in this process.

the rough devices shows very slight variation during the stress time, while that of the flat devices degrades about $15 \%$ after $1200 \mathrm{~s}$ stress. The degradation of bandgap light emission indicates the formation of interface states, which act as nonradiative recombination centers. The interface states may be induced due to the breakage of the $\mathrm{Si}-\mathrm{H}$ bonds by injected holes. The stress of hole current generates more interface states in the flat oxide than in the rough oxide. The rough oxide is more robust to tunneling carrier stress than the flat oxide, since the momentum (energy) of the injected hole tunneling from $\mathrm{Al}$ gate is scattered by the oxide roughness, and this lowers the injected hole momentum perpendicular to the interface to bombard the Si/oxide interface. The light emission of NMOS diodes showed similar results.

Fig. 5 shows the speculative mechanism of the roughness-enhanced oxide reliability in PMOS tunneling diodes. When the carriers tunnel through the oxide, they are scattered by the oxide roughness and the momentum of the injected holes perpendicular to the interface is reduced. In the PMOS tunneling diodes, the oxide degradation is related to the tunneling-hole-induced traps in bulk oxide [10], [11], and the threshold energy for the injected holes to be captured by $\mathrm{O}$ Vacancy is $1.8 \mathrm{eV}$ [18]. This indicates that only the hole with injected energy higher than $1.8 \mathrm{eV}$ can be trapped in the oxide and can induce oxide degradation. Since the reliability of the MOS tunneling diode is related to the energy of the injected holes, the perpendicular energy reduction of the holes tunneling through the oxide could improve the electrical and optical reliability of the devices. However, it is an intuitive model, and more evidence is needed to give a clear picture of the phenomenon.

\section{CONCLUSION}

The ultrathin oxide reliability is enhanced by introducing oxide roughness. The very high vacuum prebake is demonstrated to be an effective method to produce such oxide roughness. The rough oxide can be a novel technology to improve both the electrical and optical reliability of MOS devices.

\section{REFERENCES}

[1] T. Yamanaka, S. J. Fang, H.-C. Lin, J. P. Snyder, and C. R. Helms, "Correlation between inversion layer mobility and surface roughness measured by AFM," IEEE Electron Device Lett., vol. 17, pp. 178-180, Apr. 1996.

[2] J. Koga, S. Takagi, and A. Toriumi, "A comprehensive study of MOSFET electron mobility in both weak and strong inversion regimes," in IEDM Tech. Dig., 1994, pp. 475-478.

[3] T. Nakanishi, S. Kishii, and A. Ohsawa, "Degradation of time-dependent dielectric breakdown characteristics of MOS capacitors by silicon surface roughness," in VLSI Tech. Dig., 1989, pp. 79-82.

[4] C. W. Liu, M. H. Lee, M. J. Chen, C-F. Lin, and M. Y. Chern, "Roughness-enhanced electroluminescence from metal oxide silicon tunneling diodes," IEEE Electron Device Lett., vol. 21, pp. 601-603, Dec. 2000.

[5] J. W. Lyding, K. Hess, and I. C. Kizilyalli, "Reduction of hot electron degradation in metal oxide semiconductor transistors by deuterium processing," Appl. Phys. Lett., vol. 68, no. 18, pp. 2526-2528, 1996.

[6] C. G. Van de Walle and W. B. Jackson, "Comment on 'Reduction of hot electron degradation in metal oxide semiconductor transistors by deuterium processing,," Appl. Phys. Lett., vol. 69, no. 16, pp. 2441-2443, 1996.

[7] C.-H. Lin, M. H. Lee, and C. W. Liu, "Correlation between Si-H/D bond desorption and injected electron energy in metal-oxide-silicon tunneling diodes," Appl. Phys. Lett., vol. 78, no. 5, pp. 637-639, 2001.

[8] Z. Chen, K. Hess, J. Lee, J. W. Lyding, E. Rosenbaum, I. Kizilyalli, S. Chetlur, and R. Huang, "On the mechanism for interface trap generation in MOS transistors due to channel hot carrier stressing," IEEE Electron Device Lett., vol. 21, pp. 24-26, Jan. 2000.

[9] Z. Chen, P. Garg, V. Singh, and S. Chetlur, "Role of holes in the isotope effect and mechanisms for the metal-oxide-semiconductor device degradation," Appl. Phys. Lett., vol. 79, no. 2, pp. 212-214, 2001.

[10] C.-H. Lin, F. Yuan, B.-C. Hsu, and C. W. Liu, "The isotope effect of hydrogen release in the metal/oxide/n-silicon tunneling diodes," SolidState Electron., Jan. 2002, submitted for publication.

[11] K. Deguchi, S. Uno, A. Ishida, T. Hirose, Y. Kamakura, and K. Taniguchi, "Degradation of ultra-thin gate oxides accompanied by hole direct tunneling: Can we keep long-term reliability of p-MOSFETs ?," in IEDM Tech. Dig., 2000, pp. 327-330.

[12] S. Higai and T. Ohno, "Surface hydrogenation as a method to purify and flatten a silicon surface," Appl. Phys. Lett., vol. 78, no. 24, pp. 3839-3841, 2001.

[13] F. W. Smith and G. Ghidini, "Reaction of oxygen with Si(111) and (100): Critical conditions for the growth of $\mathrm{SiO}_{2}$," J. Electrochem. Soc., vol. 129, pp. 1300-1306, 1982.

[14] B.-C. Hsu, K. F. Chen, C.-C. Lai, and C. W. Liu, "Oxide roughness effect on tunneling current of MOS diodes," IEEE Trans. Electron Devices, submitted for publication.

[15] V. Tsai, X.-S. Wang, E. D. Williams, J. Schneir, and R. Dixson, "Conformal oxides on Si surfaces," Appl. Phys. Lett., vol. 71, no. 11, pp. 1495-1497, 1997.

[16] B. E. Weir, P. J. Silverman, D. Monroe, K. S. Krisch, M. A. Alam, G. B. Alers, T. W. Sorsch, G. L. Timp, F. Baumann, C. T. Liu, Y. Ma, and D. Hwang, "Ultra-thin gate dielectrics: They break down, but do they fail?," in IEDM Tech. Dig., 1997, pp. 73-76.

[17] C. W. Liu, C.-H. Lin, M. H. Lee, S. T. Chang, Y.-H. Liu, M.-J. Chen, and C. -F. Lin, "Enhanced reliability of electroluminescence from metal-oxide-silicon tunneling diodes by deuterium incorporation," Appl. Phys. Lett., vol. 78, no. 10, pp. 1397-1399, 2001.

[18] A. Yokozawa and Y. Miyamoto, "First principles exploration of possible trap terminators in $\mathrm{SiO}_{2}$," Appl. Phys. Lett., vol. 73, no. 8, pp. 1122-1124, 1998. 